\title{
Factors influencing healthy food choices of university students
}

\section{Fatores que influenciam a escolha de comida saudável nos estudantes universitários}

\author{
Bruno Sousa ${ }^{1}$, Cíntia Ferreira-Pêgo ${ }^{1}$, Catarina Alves Pacheco ${ }^{2}$, Carolina Pereira ${ }^{2}$, Mariana Batalha ${ }^{2}$, \\ Tânia Santos ${ }^{2}$, Rita Vitorino de Carvalho ${ }^{3 *}$
}

${ }^{1}$ CBIOS Lusófona's Research Center for Biosciences and Health Technologies, Av. Campo Grande 376, 1749-024 Lisbon, Portugal; ${ }^{2}$ School of Sciences and Health Technologies, Universidade Lusófona de Humanidades e Tecnologias, Av. Campo Grande 376, 1749-024 Lisbon, Portugal; ${ }^{3}$ ECEO - ULHT - Universidade Lusófona de Humanidades e Tecnologias, Av. Campo Grande 376, 1749-024 Lisbon, Portugal

*Corresponding author: rita.carvalho@ulusofona.pt

\begin{abstract}
Healthy foods should be available to anyone, especially young adults at educational institutions. University students can suffer a lack of preoccupation with their health and consequently also present unhealthy food choices, due to the adaptation of a new environment, study stress, lack of proper time management, and busy class schedules.

This study consisted of two cross-sectional observational analyzes, in which students were randomly recruited from the university, and data was obtained through the Computer-aided web interviewing survey method, through a web-based questionnaire. The sample included 584 participants, 297 from 2018 and 287 from 2019.

The preference and choice for a healthy snack bar in university facilities seem to be conditioned mainly by the price of meals, as most students mentioned this as the main condition of their choice, both in 2018 and 2019. In this sense, most respondents report that only "sometimes" would they be willing to pay more than usual or travel a longer distance, to dine in a healthier snack bar.

We conclude that the factor that most influences the choice of healthy food is the price, but there is a higher demand and consumption trend for healthy food in young college students.
\end{abstract}

Keywords: Food choice, consumption trends, healthy food, behaviors, university students

\begin{abstract}
Resumo
Alimentos saudáveis deveriam estar disponíveis para qualquer pessoa, especialmente para jovens adultos nas suas instituições de ensino. Devido à adaptação a um novo ambiente, stress provocado pelo estudo, falta de gestão adequada do tempo e horários ocupados pelas aulas, os estudantes universitários podem sofrer de falta de preocupação com a sua saúde e, consequentemente, também apresentar escolhas alimentares pouco saudáveis.

Este estudo consistiu em duas análises observacionais transversais. Os estudantes foram recrutados aleatoriamente na universidade, e os dados foram obtidos através do método de pesquisa Computer-aided web interviewing, através de um questionário enviado pela web.

A amostra final incluiu 584 participantes, sendo que 297 são procedentes da análise de 2018 e 287 de 2019.

A preferência e escolha por um bar mais saudável nas instalações universitárias, parece ser condicionada, sobretudo, pelo preço das refeições, já que a maioria dos estudantes referiu esta como a principal condicionante da sua escolha, tanto em 2018, como em 2019. Neste sentido, a maioria dos inquiridos refere que só "às vezes" estaria disposto a pagar mais do que o habitual, ou percorrer uma maior distância, para efetuar as suas refeições num bar saudável.

Concluímos que o fator que mais influencia a escolha de comida saudável é o preço, contudo parece existir uma maior procura, preocupação e tendência de consumo por comida saudável nos jovens universitários.
\end{abstract}

Palavras-chave: Escolha alimentar, tendências de consumo, comida saudável, comportamentos, estudantes universitários 


\section{Introduction}

Healthy foods, like fruits, vegetables, legumes, and nuts, among others, should be available to anyone, especially young people, for example at the school level (1). In the National Program for the Promotion of Healthy Eating, one of the action plans for 2018-2019 was the "Modification of food supply in certain public spaces (including higher education institutions)" and for 20192020: "Contribute technically to the implementation of a simplifying nutrition labeling system"(4). Thus, schools and universities should be able to encourage young people to choose higher quality food products by promoting certain menu changes (2).

Today it is known that young consumers are well informed about the products they eat (3) and also have become more aware of the foods they chose both at home and away from home (4). Increasingly interested in adhering to a healthy diet, young people are progressively opting for more complete food products, both in terms of their nutritional value, as well as their origin, the form of production, including locally grown and organically grown produce, the composition, and the healthiness of the food product $(5,6)$. Conscientious consumers value personal health and are sensitive to the effects of food products (5). Young people also place progressively greater attention on the quality of a product. In addition to the quality parameter of a food product, other factors influence young people's choices, namely economic (7), social, and personal taste (3) factors.

However, due to the adaptation of a new environment, study stress, lack of proper time management and busy class schedules, these university students can suffer a lack of concern about their own health and consequently also present unhealthy food choices $(8,9)$. The presence of healthy restaurants in the university campus could be a possible way to prevent these unhealthy patterns. Taking this into consideration, the main aim of the present study was to describe the factors that may influence the choice for a healthy restaurant in the university campus, as well as the trends in healthy food consumption among university students from the Universidade Lusófona de Humanidades e Tecnologias (ULHT) in Lisbon, Portugal.

\section{Introdução}

Uma alimentação saudável deveria estar ao alcance de qualquer pessoa, principalmente dos mais jovens, por exemplo a nível das instituições educativas (1). No Programa Nacional para a Promoção da Alimentação Saudável, um dos planos de ações para 2018-2019 é a "Modificação da oferta alimentar em determinados espaços públicos (instituições do ensino superior)" e para 2019-2020: "Contribuir tecnicamente para a implementação de um sistema de rotulagem nutricional simplificativo" (2).

Hoje em dia sabe-se que o jovem consumidor está bem informado no que toca aos produtos que ingere (3), tanto em casa como fora dela (4).

Tendo cada vez mais interesse na adesão a uma dieta saudável, os jovens optam progressivamente por produtos alimentícios mais completos, quer no que toca ao seu valor nutricional, assim como, à origem, à forma de produção do produto, incluindo produtos cultivados localmente e organicamente, à composição, e à salubridade do produto alimentício $(5,6)$. O tipo de consumidor consciente valoriza a saúde pessoal e é sensível aos efeitos dos produtos alimentícios (5). Os jovens colocam ainda, uma atenção progressivamente maior na qualidade de um produto. Além do parâmetro de qualidade de um produto alimentício, existem ainda outros fatores que influenciam as escolhas dos jovens, sendo eles fatores económicos (7), sociais e o gosto pessoal (3).

No entanto, devido à adaptação a um novo ambiente, stress provocado pelo estudo, falta de gestão adequada do tempo e horários ocupados pelas aulas, os universitários podem sofrer uma falta de preocupação com a própria saúde e, consequentemente, também apresentar escolhas alimentares pouco saudáveis $(8,9)$. A presença de restaurantes saudáveis no campus da universidade poderá ser uma forma possível de evitar esses padrões prejudiciais. Tendo em consideração todos estes fatores, o principal objetivo do presente estudo foi descrever os fatores que podem influenciar a escolha de um restaurante saudável no campus da universidade, bem como as tendências no consumo de alimentos entre estudantes universitários da Universidade Lusófona de Humanidades e Tecnologias (ULHT) em Lisboa, Portugal. 


\section{Material and Methods}

\section{Design and study population}

The present study consists of two cross-sectional observational analyses to evaluate the habits, preferences, and choices about the dining facilities existing at the university campus of the ULHT. Participants $(>18$ years old) were randomly recruited from this university, all were students from a wide range of academic courses, from architecture and engineering, to health sciences, such as nutrition, veterinary, sports or psychology. The effective sample size of the present study was 584 participants, 297 coming from the 2018 analysis and 287 coming from the cross-sectional assessment performed during 2019.

\section{Habit assessment and consumption preference}

Secondary data was searched using indexed scientific databases and a program from the Portuguese Ministry of Health. Primary data was obtained through a computer-aided web interviewing survey method, in which the respondent completed the questionnaire through a web link. Data collection was performed using a structured questionnaire, composed of four sections. In the first and second sections, the personal characteristics and the course attended were collected. The third section explored the eating habits, how daily meals were consumed, the average time to consume them, and the monetary expenditure each respondent made per day in snack bars or canteens. The fourth section looked at the trend toward eating healthy food in university snack bars, specifically, the types of foods likely to be chosen , willingness to travel further, , and the willingness to pay more for healthy options.

At the evaluated university campus facilities, the students have available three restaurants: two of them presenting traditional Portuguese food ("A Cantina" and "Xiribitátá") and one presenting healthy food options, including homemade meals, vegan, vegetarian, lower fat/carb/ processed ingredients, , etc. ("100 Corantes").

\section{Statistical analysis}

Data are presented either as means and standard deviation (SD) for continuous variables or numbers and percentages for dichotomous variables. All analyses were performed using SPSS version 26.0 software (SPSS Inc, Chicago, IL).

\section{Material e Métodos}

\section{Desenho e estudo da população}

O presente estudo consiste em duas análises observacionais transversais com o objetivo de avaliar os hábitos, preferências e escolhas sobre as instalações de restauração existentes no campus universitário da Universidade Lusófona de Humanidades e Tecnologias (ULHT), Lisboa (Portugal). Os participantes (>18 anos) foram recrutados aleatoriamente na universidade, e são estudantes dos mais variados cursos académicos, desde arquitetura, passando por engenharias e terminando nas áreas da saúde, como nutrição, veterinária, desporto ou psicologia. O tamanho efetivo da amostra do presente estudo foram 584 participantes, sendo que 297 são procedentes da análise de 2018 e 287 são procedentes da avaliação transversal realizada durante o ano 2019.

\section{Avaliação de hábitos e preferência de consumo}

Para obter o resultado do estudo, a pesquisa de dados secundários foi efetuada com recurso a base de dados científicas indexadas e programa do Ministério da Saúde de Portugal. Os dados primários foram obtidos através do método de pesquisa Computer-aided web interviewing, em que o respondente preenche o questionário através de um link enviado pela web. A recolha de dados foi realizada por meio de um questionário estruturado, composto por quatro seções. Na primeira e segunda seção, foram recolhidas as características pessoais e o curso frequentado. A terceira seção explorou os hábitos de refeição, as formas de consumo das refeições diárias, o tempo, em média, para as consumir, e o dispêndio monetário que cada respondente efetuou por dia, nos bares ou cantina. A quarta seção analisou a tendência de consumo de comida saudável nos bares da universidade, os tipos de alimentos que escolheria, se estaria disposto a percorrer uma distância maior para os consumir e a predisposição de pagar mais por este tipo de alimentos.

Nas instalações universitárias avaliadas, os alunos têm à sua disposição 3 restaurantes: 2 deles apresentando comida tradicional portuguesa ("A Cantina" e "Xiribitátá") e 1 deles apresentando opções de alimentação saudável, como por exemplo refeições caseiras, vegan, vegetariano, baixo em gordura/ hidratos de carbono/ ingredientes processados, etc. ("100 Corantes").

\section{Análise estatística}

Os dados são apresentados como médias e desvio padrão (DP) para variáveis contínuas ou números e percentagens para variáveis dicotómicas. Todas as análises 


\section{Results}

A total of 584 students, 229 of whom were men and 355 women, participated in the present analyses. The general characteristics of the population according to the years in which the surveys were conducted (2018 vs. 2019) are summarized in Table 1. Most of the individuals surveyed in 2018 were aged between 20 and 25 years $(70.40 \%)$, were women $(62.60 \%)$, Portuguese $(57.20 \%)$ and mainly from health science courses $(41.40 \%)$. Very similar results were obtained in 2019 , except for the origin of the academic course, as in this case, $49.10 \%$ of respondents were from economics, business, or engineering courses.

Table 2 shows the characterization of this population's use of catering facilities on ULHT's university campus. The number of lunches and dinners held at the same university declined in 2019 compared with 2018, however, the number of snacks and light meals increased over the same period. The largest percentage $(24.90 \%$ in 2018 vs. $34.80 \%$ in 2019 ) of students reported spending on average between 1-3€ per day in ULHT bars. Also, the foram realizadas mediante o software SPSS versão 26.0 (SPSS Inc, Chicago, IL).

\section{Resultados}

Um total de 584 estudantes da ULHT, sendo que 229 eram homens e 355 mulheres, participaram nas presentes análises. As características gerais da população, de acordo com os anos em que foram realizadas as pesquisas (2018 vs. 2019) estão resumidas na Tabela 1. Os indivíduos inquiridos no ano de 2018 apresentavam, na sua maioria (70.40\%) entre 20 e 25 anos, eram mulheres $(62.60 \%)$, portuguesas $(57.20 \%)$ e sobretudo pertencentes aos cursos de ciências da saúde (41.40\%). Resultados muito similares foram obtidos em 2019, com exceção para a procedência do curso académico, já que neste caso $49.10 \%$ dos inquiridos pertenciam a cursos de economia, gestão ou engenharias.

Na Tabela 2 pode ser observada a caracterização da utilização por parte desta população das instalações de restauração existentes no campus universitário da ULHT.

Table 1 - General characteristics of the study population categorized by year of research. Data expressed as percentages (number of respondents)

Tabela 1 -Características gerais da população caracterizadas por ano de pesquisa. Dados expressos em percentagens (número de respondentes)

\begin{tabular}{|lcc|}
\hline & $\begin{array}{c}\mathbf{2 0 1 8} \\
(\mathrm{n}=297)\end{array}$ & $\begin{array}{c}\mathbf{2 0 1 9} \\
(\mathrm{n}=287)\end{array}$ \\
\hline Age / Idade, \% (n) & $14.50(43)$ & $19.50(56)$ \\
$<20$ years / $<20$ anos & $70.40(209)$ & $54.70(157)$ \\
$20-25(2018), 20-24(2019)$ years / anos & $11.40(34)$ & $18.80(54)$ \\
$26-30(2018), 25-30(2019)$ years / anos & $3.70(11)$ & $7.0(20)$ \\
$>30$ years / >30 anos & & \\
& & $41.10(118)$ \\
Gender / Sexo, \% (n) & $37.40(111)$ & $58.90(169)$ \\
Men / Homem & $62.60(186)$ & \\
Women / Mulher & & $77.00(221)$ \\
& & $13.60(39)$ \\
Nationality / Nacionalidade, \% (n) & $57.20(170)$ & $9.40(27)$ \\
Portuguese / Portuguesa & $31.00(92)$ & \\
Foreign / Estrangeiro & $11.80(35)$ & \\
Dual nationality / Dupla nacionalidade & & $36.20(104)$ \\
& & $2.80(8)$ \\
Academic course / Curso académico, \% (n) & $41.40(123)$ & $49.10(141)$ \\
Health / Saúde & $9.10(27)$ & $9.10(26)$ \\
Arts / Artes & $14.50(43)$ & \\
Economics / Economia & $31.30(93)$ & \\
Letters / Humanidades & & \\
\end{tabular}


Table 2 - Characterization of the university facilities utilization. Data expressed as percentages (number of respondentes) Abbreviations: ULHT, Universidade Lusófona de Humanidades e Tecnologias

Tabela 2 - Caracterização da utilização das instalações da universidade. Dados expressos em percentagens (número de respondentes). Abreviaturas: ULHT, Universidade Lusófona de Humanidades e Tecnologias

\begin{tabular}{|c|c|c|}
\hline . & $\begin{array}{c}2018 \\
(n=297)\end{array}$ & $\begin{array}{c}2019 \\
(n=287)\end{array}$ \\
\hline $\begin{array}{l}\text { Days per week aftending UL.HT / Dias por semana } \\
\text { que frequenta ULHT }\end{array}$ & 4.05 (1.49) & $4.28(1.16)$ \\
\hline $\begin{array}{l}\text { Hears per day attending ULHT / Heras por dia que } \\
\text { passa na ULHT }\end{array}$ & - & $2.04(0.75)$ \\
\hline $\begin{array}{l}\text { Lanches and Dinners eaten at UL.HT / Almoços e } \\
\text { jantares por semana realizados na ULHT }\end{array}$ & 1.65 (1.59) & $1.46(1.74)$ \\
\hline $\begin{array}{l}\text { Light meals per week eaten at ULHT / Refeicôes } \\
\text { ligeiras por semana realizadas ma UL.HT }\end{array}$ & $1.97(1.66)$ & $2.34(2.27)$ \\
\hline of & $13.80(41)$ & $15.30(44)$ \\
\hline $1-3 E$ & $24.90(74)$ & $34.80(100)$ \\
\hline $3-5 \epsilon$ & $29.00(86)$ & $25.40(\mathrm{T3})$ \\
\hline $5-7 e$ & $24.20(72)$ & $19.20(55)$ \\
\hline$>7 €$ & $8.10(24)$ & $5.20(15)$ \\
\hline \multicolumn{3}{|c|}{ When not having lunch or dinner at ULHT bars? / Quando não almoça ou janta nos bares da ULFT?, \% ( $n$ ) } \\
\hline Nho consuma No cossumption & $16.20(48)$ & $5.20(15)$ \\
\hline Trago ou coesumo $\mathrm{cm}$ casa/ I bring or eat at hume & $49.20(146)$ & $77.40(222)$ \\
\hline Foea da ULHT/ Outside ULHT & $34,70(103)$ & $17,40(50)$ \\
\hline \multicolumn{3}{|c|}{ When not having light meals at ULHT bars? / Quando não lancha nos bares da ULHT?, \% (n) } \\
\hline Nio consumo/ No consumption & $20.20(60)$ & $8.70(25)$ \\
\hline Trago ou coesumo $\mathrm{cm}$ casa/ I bring or eat at home & $51.90(154)$ & $75.30(216)$ \\
\hline Foea da ULHT/ Outside UL.HT & $27.90(83)$ & $16,00(46)$ \\
\hline \multicolumn{3}{|c|}{ How much time for lunch at ULHT2 / Quanto tempo de almoço ma ULHT?, \% (n) } \\
\hline No coesumption / Nho consumo & $14.80(44)$ & $17,40(50)$ \\
\hline Up to 10 minutes / Até 10 mirnutos & $11.40(34)$ & $16.00(46)$ \\
\hline $10-20$ minutes / $10-20$ minutcos & $27.30(81)$ & $13.20(38)$ \\
\hline 20-30 minutes $/ 20-30$ minutos & $24.20(72)$ & $19.50(56)$ \\
\hline$>30$ minutes $/>30$ minuinas & $18.50(55)$ & $33.80(97)$ \\
\hline \multicolumn{3}{|c|}{ How much time for dinner at UL.HT2 / Quanto tempo de jantar na ULHT?, \% (n) } \\
\hline No ecesumption / Nho consumo & $59.90(178)$ & $82.60(237)$ \\
\hline Up to 10 minutes / Até 10 mimutos & $4.70(14)$ & $6.30(18)$ \\
\hline 10-20 minutes / $10-20$ minutos & $8.10(24)$ & $3.50(10)$ \\
\hline 20-30 minutes / $20-30$ minutos & $3.70(23)$ & $3.50(10)$ \\
\hline$>30$ minutes $/>30$ minutas & $4.40(13)$ & $4.20(12)$ \\
\hline \multicolumn{3}{|c|}{ How much time for light meals at ULHT? / Quanto tempe de refeiçồs lig̣eiras na ULHT?, \% (n) } \\
\hline No consumption / Nono consumo & $11.40(34)$ & $24.40(70)$ \\
\hline Up to 10 minutes $/$ Até 10 minutes & $55.60(165)$ & $52.60(151)$ \\
\hline $10-20$ minutes $/ 10-20$ minutos & $21.20(63)$ & $13.90(40)$ \\
\hline 20-30 minutes / $20-30$ minutos & $4.70(14)$ & $5.20(15)$ \\
\hline$>30$ minutes $/>30$ minunos & $2.40(7)$ & $3.80(11)$ \\
\hline \multicolumn{3}{|c|}{ How much time for breakfast at UL.HT? / Quante tempo de pequeno-almoço na ULHT?, \% (n) } \\
\hline No consumption / Nifo consumo & - & $49.50(142)$ \\
\hline Up to 10 minutes / Até 10 minutos & . & $33.80(97)$ \\
\hline $10-20$ minues $/ 10-20$ minutos & - & $10.80(31)$ \\
\hline 20-30 minutes / $20-30$ minutcs & . & $3.50(10)$ \\
\hline$>30$ minutes / $>30$ minunos & - & $2.40(7)$ \\
\hline
\end{tabular}


majority of the participants reported consuming at or bringing home their lunches, dinners and light snacks when they are at ULHT and do not use their bars. Consumption in other bars/restaurants outside of ULHT decreased almost by half, compared to lunch/dinner (34.70\% in 2018 vs. $17.40 \%$ in 2019 ) as well as snacks and light meals (27.90\% in 2018 vs. $16.00 \%$ in 2019). Regarding preferences or opinions about the existing catering facilities on the Lisbon university campus (Table 3) during 2018, most students have no preference over which snack bar is the cheapest, however, it seems evident that "A Cantina" is their favorite, as $41.40 \%$ consider the best value for money, $35.00 \%$ think it has the best distance and $33.30 \%$ think it has the best environment, compared to the other options. During 2019,
O número de almoços e jantares realizados na mesma universidade decresceu em 2019, em comparação com 2018, contudo, o número de lanches e refeições ligeiras aumentou no mesmo período de tempo. A maior percentagem (24.90\% em 2018 vs. $34.80 \%$ em 2019) dos alunos refere gastar em média, entre 1 a $3 €$ por dia, nos bares da ULHT. Também a maioria da amostra refere consumir em/ou trazer de casa, os seus almoços, jantares e refeições ligeiras quando está na ULHT e não utiliza os seus bares. De referir que o consumo em outros bares/restaurantes fora da ULHT diminuiu quase para metade, em relação a almoços/ jantares $(34.70 \%$ em 2018 vs. $17.40 \%$ em 2019) assim como, lanches e refeições ligeiras $(27.90 \%$ em 2018 vs. $16.00 \%$ em 2019).

Table 3 - University snack bar preferences. Data expressed as percentages (number of respondentes) Tabela 3 - Preferências sobre os bares da universidade. Dados expressos em percentagens (número de respondentes).

\begin{tabular}{|c|c|c|}
\hline & $\begin{array}{c}2018 \\
(n=297)\end{array}$ & $\begin{array}{c}2019 \\
(n=287)\end{array}$ \\
\hline \multicolumn{3}{|c|}{ Cheapest snack bar / Bar mais económico, \% (n) } \\
\hline No preference / Sem preferência & $37.40(111)$ & - \\
\hline Xüribitata & $32.30(96)$ & - \\
\hline A Cantina & $25.60(76)$ & - \\
\hline \multicolumn{3}{|c|}{ Best quality-price snack bar / Bar melhor qualidade-preço, \% (n) } \\
\hline No preference / Sem preferêneia & $29.00(86)$ & - \\
\hline Xiribitata & $21.20(63)$ & - \\
\hline A Cantina & $41.40(123)$ & - \\
\hline \multicolumn{3}{|c|}{ Best distance snack bar / Bar melhor distância, \% (n) } \\
\hline No preference / Sem preferência & $30.00(89)$ & - \\
\hline Xiribitata & $23.20(69)$ & - \\
\hline A Cantina & $35.00(104)$ & - \\
\hline \multicolumn{3}{|c|}{ Best environment snack bar / Bar melhor ambiente, \% (n) } \\
\hline No preference / Sem preferência & $28.60(85)$ & - \\
\hline Xiribitata & $29.00(86)$ & - \\
\hline A Cantina & $33.30(99)$ & $=$ \\
\hline \multicolumn{3}{|c|}{ Opinion about "Xiribitata"? / Opinião sobre "Xiribitata"?, \% (n) } \\
\hline Do not like / Não gosto & $\cdot$ & $12.50(36)$ \\
\hline Do not go / Não vou & - & $5.60(16)$ \\
\hline Indifferent / Indiferente & - & $35.90(103)$ \\
\hline Like / Gosto & $\cdot$ & $46.00(132)$ \\
\hline \multicolumn{3}{|c|}{ Opinion about "A Cantina"? / Opiniāo sobre "A Cantina"?, \% (n) } \\
\hline Do not like / Não gosto & $\cdot$ & $4.90(14)$ \\
\hline Do not go / Não vou & $\cdot$ & $8.00(23)$ \\
\hline Indifferent/ Indiferente & - & $27.20(78)$ \\
\hline Like / Gosto & $\cdot$ & $59.90(172)$ \\
\hline \multicolumn{3}{|c|}{ Opinion about "100 Corantes"? / Opinião sobre "100 Corantes"?, \% (n) } \\
\hline Do not like / Não gosto & $\cdot$ & $2.10(6)$ \\
\hline Do not go / Não vou & - & $35.50(102)$ \\
\hline Indifferent / Indiferente & - & $24.40(70)$ \\
\hline Like / Gosto & - & $38.00(109)$ \\
\hline
\end{tabular}


the trend seems to continue, as $59.90 \%$ say they like "A Cantina", while only $46.00 \%$ have the same opinion for "Xiribitatá". Finally, $38.00 \%$ think that "100 Corantes" presents a concept of healthier food, however, it is a bit more expensive and in a location slightly farther from the center of the campus.

Regarding the preference and choice for a healthier bar in ULHT facilities, it seems that this decision is mainly conditioned by the price of meals, as most students mentioned this as the main condition of their choice, both in 2018 as in 2019. In this sense, most respondents also point out that only "sometimes" would they be willing to pay more than usual, or to go further, to dine in a healthier snack bar. Finally, $79.40 \%$ and $72.80 \%$ of respondents were interested in having access to the
Em relação às preferências ou opiniões sobre as instalações de restauração existentes no campus de Lisboa (Tabela 3) durante o ano de 2018, os alunos, na sua maioria, não têm preferência sobre qual o bar mais económico, contudo, parece evidente que A Cantina é o bar preferido dos mesmos, já que $41.40 \%$ considera que apresenta a melhor qualidade-preço, $35.00 \%$ considera que se encontra a uma melhor distância e 33.30\% refere o seu melhor ambiente, em comparação com as restantes opções. Durante o ano de 2019, a tendência parece manter-se, já que $59.90 \%$ refere gostar d'A Cantina, enquanto somente $46.00 \%$ têm a mesma opinião em relação ao Xiribitatá e $38.00 \%$ em relação ao 100 corantes, que apresenta um conceito de comida mais saudável, um pouco mais dispendioso e com uma lo-

Table 4 - University healthy snack bar preferences and choices. Data expressed as percentages (number of respondentes)

Tabela 4 - University healthy snack bar preferences and choices. Dados expressos em percentagens (número de respondentes).

\begin{tabular}{|c|c|c|}
\hline & $\begin{array}{c}2018 \\
(\mathrm{n}=297)\end{array}$ & $\begin{array}{c}2019 \\
(\mathrm{n}=287)\end{array}$ \\
\hline \multicolumn{3}{|c|}{ Would you be a customer of a healthy snack bar at ULHT? / Seria cliente de um bar saudável na ULHT, \% (n) } \\
\hline Never consume / Nunca consumiria & $1.00(3)$ & $2.80(8)$ \\
\hline Always consume / Consumiria sempre & $17.50(52)$ & $33.80(97)$ \\
\hline Depends on the price / Depende do preço & $47.80(142)$ & $56.40(162)$ \\
\hline Depends on the product / Depende do produto & $33.70(100)$ & $7.0(20)$ \\
\hline \multicolumn{3}{|c|}{$\begin{array}{l}\text { Would you be willing to pay more at a healthier snack bar than you usually do?, \% (n) / Estaria disposto a pagar } \\
\text { mais num bar saudável do que paga habitualmente?, \% (n) }\end{array}$} \\
\hline Never / Nunca & $12.50(37)$ & $31.40(90)$ \\
\hline Rarely / Raramente & $21.50(64)$ & $0.00(0)$ \\
\hline Sometimes / Às vezes & $50.80(151)$ & $41.80(120)$ \\
\hline Always / Sempre & $13.80(41)$ & $26.80(77)$ \\
\hline \multicolumn{3}{|c|}{$\begin{array}{l}\text { Would you be a client of a healthy snack bar even traveling further?, } \% \text { (n) / Seria cliente de um bar saudável } \\
\text { mesmo percorrendo uma maior distância?, } \% \text { (n) }\end{array}$} \\
\hline Never / Nunca & $3.40(10)$ & $8.0(23)$ \\
\hline Rarely / Raramente & $13.10(39)$ & $18.10(52)$ \\
\hline Sometimes / Às vezes & $69.70(207)$ & $68.30(196)$ \\
\hline Always / Sempre & $10.80(32)$ & $5.60(16)$ \\
\hline \multicolumn{3}{|c|}{$\begin{array}{l}\text { Would you like to have access to the description of the ingredients used on the available products?, \% (n) / Gostari } \\
\text { de ter acesso à descrição dos ingredientes dos produtos disponibilizados?, \% (n) }\end{array}$} \\
\hline Yes / Sim & - & $79.40(228)$ \\
\hline No/Não/ & - & $4.90(14)$ \\
\hline Indifferent / Indiferente & - & $15.70(45)$ \\
\hline \multicolumn{3}{|c|}{$\begin{array}{l}\text { Would you like to have access to the nutritional composition of the available products?, \% (n) / Gostaria de ter } \\
\text { acesso à informação nutricional dos produtos disponibilizados?, \% (n) }\end{array}$} \\
\hline Yes / Sim & - & $72.80(209)$ \\
\hline No / Não & - & $3.10(9)$ \\
\hline Indifferent / Indiferente & - & $24.00(69)$ \\
\hline
\end{tabular}


list of products/ingredients used, as well as nutritional information, respectively. These results can be clearly seen in Table 4.

\section{Discussion}

The purpose of this study was trying to understand the trends of healthy food consumption in young Portuguese university students. The number of lunches and dinners held in ULHT snack bars/restaurants decreased in 2019 compared to 2018. However, the number of snacks and light meals increased in the same period. This denotes a tendency of respondents to adhere to fast meals, as can be observed in the scientific literature (10). Another topic to take into account is that most respondents spend on average between 1-3€/day at ULHT snack bars.

According to the literature review (2), which advocates the "Modification of food supply in certain public spaces (higher education institutions)", a healthier snack bar was created at ULHT in 2019. Related with the price to pay for having this healthy snack bar, most students indicated that only "sometimes" would be willing to pay more than usual, or to travel a greater distance inside the university, to make their meals in this restaurant. These results were also recognized by Jiumpanyarach (7), which points to economic factors as an aspect that influences young people's choices beyond the quality of the food product.

One of the action plans for 2019-2020 (2) is to contribute to the implementation of an expressive nutrition labeling system. This action is of interest to the students, as $79.40 \%$ say they have an interest in the description of products and ingredients and $72.80 \%$ would like to have access to nutritional information.

It should be noted that consumption outside of ULHT was almost halved compared to lunch/dinners, snacks and light meals from 2018 to 2019, the opening year of the "100 Corantes" snack bar. The explanation to this relation may be because this new healthy snack bar has met a need for some students, however, additional studies are needed to answer this and other remaining questions. calização ligeiramente mais afastada do centro do Campus.

Em relação à preferência e escolha por um bar mais saudável nas instalações da ULHT, parece ser que essa decisão é condicionada, sobretudo, pelo preço das refeições, já que a maioria dos estudantes referiu esta como a principal condicionante da sua escolha, tanto em 2018, como em 2019. Neste sentido, também a maioria dos inquiridos refere que só "às vezes" estaria disposto a pagar mais do que o habitual, ou percorrer uma maior distância, para efetuar as suas refeições num bar saudável. Finalmente, $79.40 \%$ e $72.80 \%$ dos inquiridos demonstram ter interesse sobre a descrição dos produtos e ingredientes disponibilizados, assim como, ter acesso à informação nutricional dos mesmos, respetivamente. Estes resultados podem ser observados em maior profundidade na Tabela 4.

\section{Discussão}

O propósito deste estudo visa entender as tendências de consumo de comida saudável em jovens universitários Portugueses. O número de almoços e jantares realizados nos bares/restaurantes da ULHT, decresceu em 2019, em comparação com 2018. Contudo, o número de lanches e refeições ligeiras aumentou no mesmo período de tempo. Isto denota uma tendência dos inquiridos em aderir a refeições rápidas, como se anteriormente descrito na evidência científica (12).

De acordo com a revisão da literatura (4), em que se defende a "Modificação da oferta alimentar em determinados espaços públicos (instituições do ensino superior)", foi criado na ULHT, em 2019, um bar com alimentação mais saudável. No entanto, a escolha dos estudantes por este tipo de alimentação revelou estar condicionada ao preço das refeições, já que a maioria dos estudantes acrescenta que só "às vezes" estaria disposto a pagar mais do que o habitual, ou a percorrer uma distância maior no interior da universidade, para efetuar as suas refeições num bar saudável. Estes resultados são reconhecidos no estudo de Jiumpanyarach, (11), que aponta os fatores económicos como um aspeto que influencia as escolhas dos jovens, para além da qualidade do produto alimentício. Outro aspeto a considerar é que a maioria dos respondentes gasta, em média entre 1-3€ por dia, nos bares da ULHT.

Um dos planos de ações para 2019-20 (4) é contribuir para a implementação de um sistema de rotulagem nutricional expressivo. E esta ação vai ao encontro do interesse dos estudantes, na medida em que $79.40 \%$ revelam ter interesse na descrição dos produtos e ingredientes utilizados na preparação dos pratos e $72.80 \%$ 


\section{Conclusions}

To the best of our knowledge, the present study is the first to describe that the number of lunches and dinners held in ULHT restaurants decreased in 2019 compared to 2018. However, the number of snacks and light meals increased in the same period. It should be noted that consumption outside of ULHT has almost halved compared to lunch/dinners, snacks and light meals from 2018 to 2019, the opening year of the healthier snack bar. Related with the price to pay for eating in this healthy restaurant, most students add that only "sometimes" would be willing to pay more than usual, or to travel a greater distance inside the university, to make their meals in this restaurant. Finally, $79.40 \%$ of the evaluated students described interest in having the description of products and ingredients and $72.80 \%$ of them would like to have access to their nutritional information.

\section{Acknowledgments}

The authors would like to acknowledge the Economics - Operational Marketing discipline, for collecting the data.

\section{Conflict of interests}

The authors report no conflicts of interest. gostariam de ter acesso à informação nutricional dos mesmos.

Importa referir que o consumo fora da ULHT, diminuiu quase para metade, em relação a almoços/jantares, lanches e refeições ligeiras, de 2018 para 2019, ano de abertura do Bar "100 Corantes". Esta relação observada poderá ser explicada pelo facto deste bar ter vindo colmatar algumas necessidades dos estudantes, contudo futuros estudos são necessários para esclarecer esta e outras questões pendentes.

\section{Conclusões}

Pelo melhor do nosso conhecimento, o presente estudo é o primeiro a descrever que o número de almoços e jantares realizados nos restaurantes da ULHT diminuiu em 2019 em comparação a 2018. No entanto, o número de lanches e refeições leves aumentou no mesmo período. É importante referir também, que o consumo de almoços e jantares, lanches e refeições leves fora da ULHT caiu quase para metade em 2019 (o ano da abertura do restaurante saudável) em comparação com 2018. Relacionado com o preço a pagar no restaurante saudável, a maioria dos estudantes acrescenta que apenas "às vezes" estaria disposto a pagar mais do que o habitual, ou a percorrer uma distância maior dentro da universidade, para efetuar as suas refeições neste estabelecimento. Finalmente, $79,40 \%$ dos estudantes avaliados descreveram interesse em ter a descrição de produtos e ingredientes utilizados na confeção dos pratos e $72,80 \%$ deles gostariam de ter acesso às suas informações nutricionais.

\section{Agradecimentos}

Os autores agradecem aos alunos da licenciatura em Economia - disciplina de Marketing Operacional, pela recolha dos dados.

\section{Conflito de interesses}

Os autores declaram não ter conflitos de interesse. 


\section{References / Referências}

1. Scalvedi ML, Saba A. Exploring local and organic food consumption in a holistic sustainability view. Br Food J. 2018;120(4):749-62.

2. Direção Geral da Saúde. Programana Nacional para a Promoção da Alimentação Saudável. Direção Geral da Saúde. Lisbon, Portugal; 2019.

3. Savelli E, Murmura F, Liberatore L, Casolani N, Bravi L. Food habits and attitudes towards food quality among young students. Int J Qual Serv Sci. 2017;9(3-4):456-68

4. Din N, Zahari MSM, Shariff SM. Customer Perception on Nutritional Information in Restaurant Menu. Procedia - Soc Behav Sci. 2012;42:413-21.

5. Jin N (Paul), Line ND, Lee SM. The health conscious restaurant consumer: Understanding the experiential and behavioral effects of health concern. Int J Contemp Hosp Manag. 2017;29(8):2103-20.

6. Bello Acebrón L, Calvo Dopico D. The importance of intrinsic and extrinsic cues to expected and experienced quality: An empirical application for beef. Food Qual Prefer. 2000;11(3):229-38.

7. Jiumpanyarach W. The impact of social trends: teenagers' attitudes for organic food market in Thailand. Int J Soc Econ. 2018 Feb 23;00-00.

8. King KA, Mohl K, Bernard AL, Vidourek RA. Does Involvement in Healthy Eating Among University Students Differ Based on Exercise Status and Reasons for Exercise?. Vol. 5, Californian Journal of Health Promotion. 2007.

9. Ganasegeran K, Al-Dubai SAR, Qureshi AM, Al-abed AAA, Am R, Aljunid SM. Social and psychological factors affecting eating habits among university students in a Malaysian medical school: a cross-sectional study. Nutr J. 2012 Jul 18;11:48.

10. Mathur S and PK Patodiya. Global perspective of fast-food consumption. Indian J Manag Sci. 2016;6(2):2249. 